\title{
Nonuniform Overlapping Method in Designing Microstrip Patch Antennas Using Genetic Algorithm Optimization
}

\author{
J. M. Jeevani W. Jayasinghe, ${ }^{1}$ Jaume Anguera, ${ }^{2,3}$ \\ Disala N. Uduwawala, ${ }^{4}$ and Aurora Andújar ${ }^{2}$ \\ ${ }^{1}$ Department of Electronics, Wayamba University of Sri Lanka, Kuliyapitiya, Sri Lanka \\ ${ }^{2}$ Technology and Intellectual Property Rights Department, Fractus, Barcelona, Spain \\ ${ }^{3}$ Electronics and Communications Department, Universitat Ramon LLull, Barcelona, Spain \\ ${ }^{4}$ Department of Electrical and Electronic Engineering, University of Peradeniya, Peradeniya, Sri Lanka
}

Correspondence should be addressed to J. M. Jeevani W. Jayasinghe; jeevani98@yahoo.com

Received 12 August 2014; Revised 17 December 2014; Accepted 18 December 2014

Academic Editor: Chih-Hua Chang

Copyright (c) 2015 J. M. Jeevani W. Jayasinghe et al. This is an open access article distributed under the Creative Commons Attribution License, which permits unrestricted use, distribution, and reproduction in any medium, provided the original work is properly cited.

\begin{abstract}
Genetic algorithm (GA) has been a popular optimization technique used for performance improvement of microstrip patch antennas (MPAs). When using GA, the patch geometry is optimized by dividing the patch area into small rectangular cells. This has an inherent problem of adjacent cells being connected to each other with infinitesimal connections, which may not be achievable in practice due to fabrication tolerances in chemical etching. As a solution, this paper presents a novel method of dividing the patch area into cells with nonuniform overlaps. The optimized design, which is obtained by using fixed overlap sizes, shows a quad-band performance covering GSM1800, GSM1900, LTE2300, and Bluetooth bands. In contrast, use of nonuniform overlap sizes leads to obtaining a pentaband design covering GSM1800, GSM1900, UMTS, LTE2300, and Bluetooth bandswith fractional bands with of $38 \%$ due to the extra design flexibility.
\end{abstract}

\section{Introduction}

Genetic algorithm (GA) is a powerful optimization technique that has been shown to be useful in a wide area of electromagnetics such as antennas, antenna arrays, and radar systems $[1,2]$. Use of GA optimization to obtain desired radiation parameters by means of designing a set of metallic strips was discussed in [2], nearly 20 years ago. Optimization of several parameters, such as patch shape and ground shape, to design broadband [3-6], multiband [6-11], miniature [12], and highdirectivity [13] microstrip patch antennas (MPAs) using GA is presented in the literature.

Optimization of patch shape, having random slots in the patch area, provides several resonant current paths. As a result, the MPA resonates at several frequencies. Therefore, GA optimization has been used by researchers to design multiband MPAs. If the dimensions are selected such that these frequencies are close to each other, their resonant bands can overlap to give an increased single bandwidth to the MPA. In case of a GA modified patch shape, a longer current path can be created and the effective electrical length becomes larger compared to classical patches, where the current flows along a straight line. In this sense, shape optimization of the patch has been used to design miniature MPAs.

The basic method in optimization of patch shape is the use of traditional on/off building blocks to make cells contacting by an infinitesimal point $[1,5,9,12]$. However, it may pose a connection problem when manufacturing the microstrip patch, due to the tolerances of the chemical etching. Therefore, some overlapping methods have been suggested to avoid having cells contacting by an infinitesimal point. In [6], amorphous shapes have been created using ellipses generated by GA. In $[4,11,13]$, overlapping between neighboring cells is illustrated.

In contrast, a simple overlapping method based on shifting of the cells parallel to patch width is proposed in 


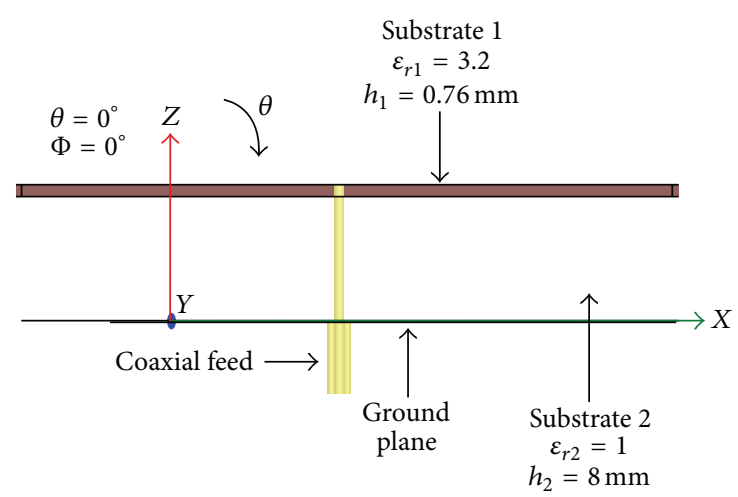

(a)

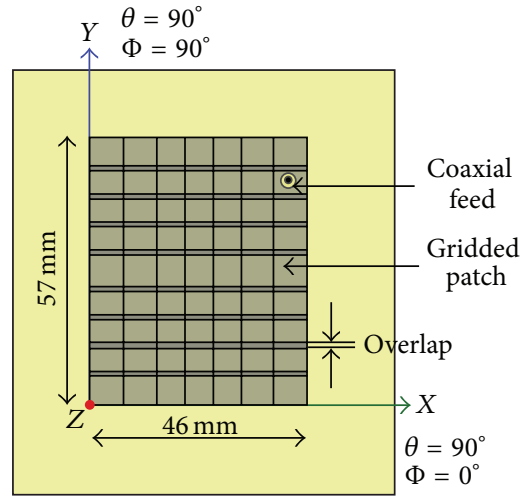

(b)

FIGURE 1: Antenna configuration. (a) Lateral view of the antenna. (b) Patch fragmented into 63 cells.

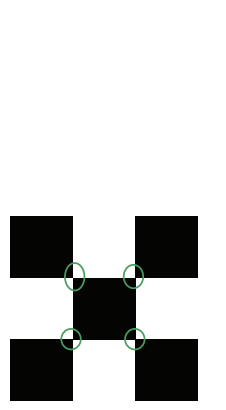

(a)

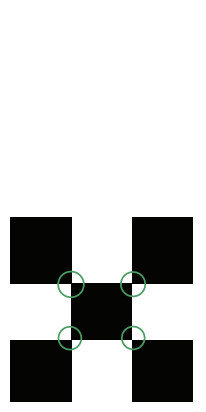

(b)

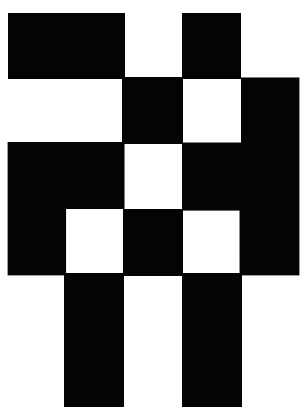

(c)

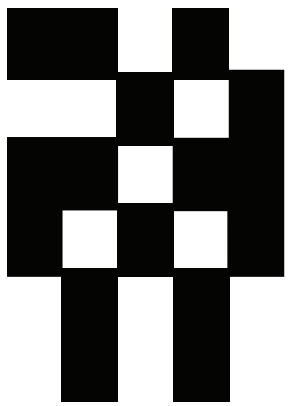

(d)

Figure 2: (a) Traditional on/off building blocks with infinitesimal connections. (b) Scheme with overlaps. (c) A possible structure with infinitesimal connections. (d) A possible structure with overlaps.

this paper. The optimized MPA designed by using uniform overlap method shows a quad-band performance covering GSM1800, GSM1900, LTE2300, and Bluetooth bands. Further, use of nonuniform overlap sizes leads to obtaining a pentaband design including UMTS band too. This paper consists of five sections, where Section 2 explains the antenna configuration and GA procedure; Section 3 compares the performance of the optimized designs using patch geometry and reflection coefficient plots; Section 4 analyses the results; and finally Section 5 summarizes the paper.

\section{Antenna Configuration and GA Procedure}

Neltec NX9320 (IM) (tm) which has a relative permittivity of 3.2 and a loss tangent of 0.0025 is used as the substrate for MPAs. The patch printed on the substrate suspends above a ground plane having an air gap of $8 \mathrm{~mm}$ (Figure 1(a)). All MPAs are designed on a rectangular footprint of length $46 \mathrm{~mm}$ and width $57 \mathrm{~mm}$ (Figure 1(b)). The patch size is comparable with the size of a rectangular shaped patch resonating in its fundamental mode around $2200 \mathrm{MHz}$. A $50 \Omega$ coaxial cable is used to feed the antenna.

In the GA procedure, 63 bits are used to define the patch geometry, by assigning conducting or nonconducting properties to each cell. As there are only two possible values, binary coding is used. Another five genes of the chromosome are used to define the feed position on the patch. Moreover, asymmetric patch geometries are allowed in the optimization, as it gives more flexibility to the GA to find a solution.

The traditional on/off building blocks method with infinitesimal connections is compared with a scheme consisting of overlaps (Figure 2). In the proposed scheme, overlapping based on shifting of the cell following the vertical axis is used to avoid having cells contacting by an infinitesimal point. Though there is no patch overlap parallel to patch length, cells neighboring diagonally are contacted. At this point, the question arises: what should be the width of the overlapping? Therefore, an experiment was conducted to identify the most suitable size of overlap as described in the next section.

The performance needed to achieve is broadband or multiband performance covering the GSM1800, GSM1900, UMTS, LTE2300, and Bluetooth bands. Therefore, the fitness function is defined as the summation of reflection coefficient values taken at $10 \mathrm{MHz}$ intervals ranging from $f_{1}=1710 \mathrm{MHz}$ to $f_{2}=2170 \mathrm{MHz}$ and from $f_{3}=2300 \mathrm{MHz}$ to $f_{4}=2480 \mathrm{MHz}$. The fitness function $F$ which is maximized in the search for the optimum solution is written as

$$
F=\frac{-\left(\sum_{f_{i}=f_{1}}^{f_{2}} L\left(f_{i}\right)+\sum_{f_{i}=f_{3}}^{f_{4}} L\left(f_{i}\right)\right)}{10 \cdot N},
$$




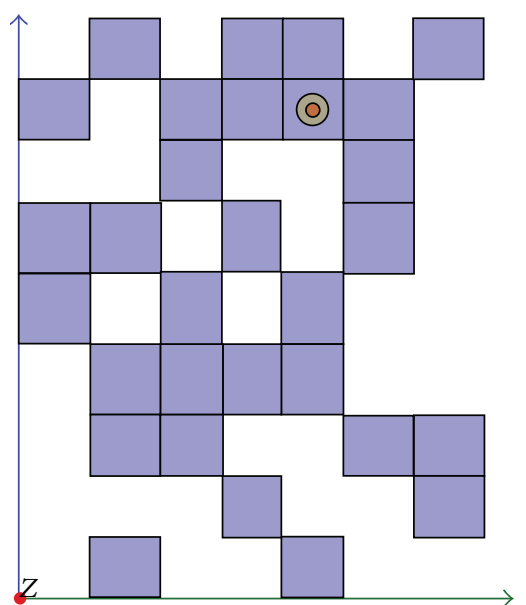

(a)

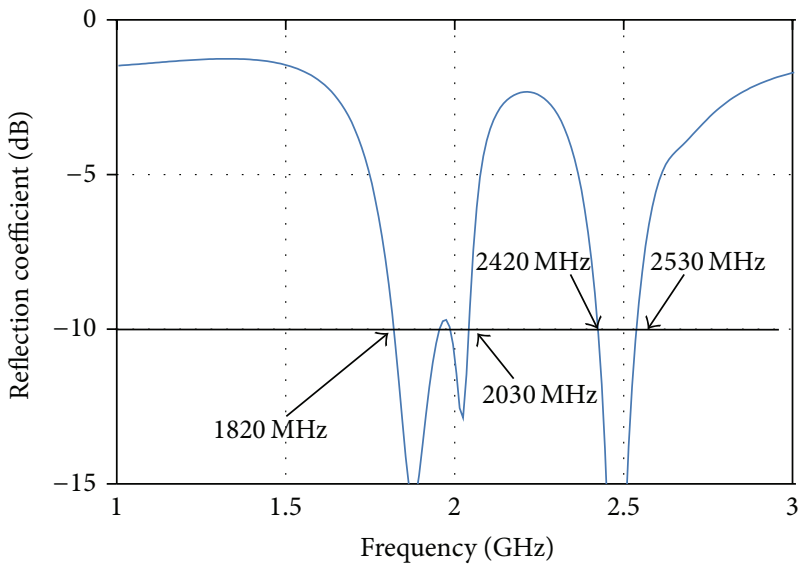

(b)

FIGURE 3: The optimized MPA with infinitesimal connections. (a) Optimized design. (b) Reflection coefficient.

where $N$ is the total number of samples $(N=65)$ and $L\left(f_{i}\right)$ is defined as

$$
L\left(f_{i}\right)= \begin{cases}\rho\left(f_{i}\right)_{\mathrm{dB}} & \rho\left(f_{i}\right)_{\mathrm{dB}} \geq-10 \mathrm{~dB} \\ -10 \mathrm{~dB} & \rho\left(f_{i}\right)_{\mathrm{dB}}<-10 \mathrm{~dB}\end{cases}
$$

where $\rho\left(f_{i}\right)_{\mathrm{dB}}$ is the reflection coefficient in $\mathrm{dB}$ at frequency $f_{i}$. The fitness function is defined in such a way that broadband solutions are preferred instead of narrowband solutions with very low reflection coefficient values. The maximum value of $F$ is unity and it is taken as the termination criterion of the optimization procedure. The fitness function can be modified to control more characteristics of the MPA such as the radiation patterns and polarization properties as and when required.

\section{Performance of the Optimized Designs}

MPAs with infinitesimal connections and three different overlap sizes between adjacent cells are examined to obtain multiband performance. Different overlap sizes have been tested to identify the most suitable size for performance improvement of the MPA. The overlap size should be large enough to be compatible for a chemical fabrication process and to contact neighboring cells properly. On the other hand, the overlap size should be small enough to have nonconducting regions on the patch area. Therefore, three different overlap sizes $(0.5 \mathrm{~mm}, 1 \mathrm{~mm}$, and $2 \mathrm{~mm})$ have been investigated for a cell size of approximately $7 \times 7 \mathrm{~mm}^{2}$. Further, an optimization is done using the novel nonuniform overlapping scheme.

3.1. Infinitesimal Connections. Initially, an MPA with infinitesimal connections between adjacent cells is optimized for comparison purposes. It operates from $1820 \mathrm{MHz}$ to $1950 \mathrm{MHz}$, from $1990 \mathrm{MHz}$ to $2030 \mathrm{MHz}$, and from $2420 \mathrm{MHz}$ to $2530 \mathrm{MHz}$ covering GSM1900 and Bluetooth partially (Figure 3 ). Though it shows multiband performance unlike classical rectangular patches, the performance is far away from the specified target.

3.2. Uniform Overlaps of $0.5 \mathrm{~mm}, 1 \mathrm{~mm}$, and $2 \mathrm{~mm}$. The optimized MPA, with an overlap of $0.5 \mathrm{~mm}$, exhibits dual resonance from $1810 \mathrm{MHz}$ to $1950 \mathrm{MHz}$ and from $2300 \mathrm{MHz}$ to $2490 \mathrm{MHz}$ (Figures 4(a) and 4(d)). Therefore, this MPA is suitable for LTE2300 and Bluetooth applications. Further, it covers GSM1800 and GSM1900 partially. However, it does not achieve the target bandwidth. The optimized MPA with overlap of $1 \mathrm{~mm}$ resonates from $1710 \mathrm{MHz}$ to $2130 \mathrm{MHz}$ and from $2290 \mathrm{MHz}$ to $2490 \mathrm{MHz}$ (Figures 4(b) and 4(d)). The bandwidth of the lower resonant frequency is larger and this MPA shows quad-band performance covering GSM1800, GSM1900, LTE2300, and Bluetooth bands. This design also does not reach the target solution. When the overlap is increased to $2 \mathrm{~mm}$, the optimized MPA resonates from $1710 \mathrm{MHz}$ to $1960 \mathrm{MHz}$ and from $2280 \mathrm{MHz}$ to $2470 \mathrm{MHz}$ (Figures 4(c) and 4(d)). It covers GSM1800 and LTE2300 bands completely and GSM1900 and Bluetooth bands partially showing a lower performance than earlier.

Bandwidth performances of the optimized designs show that the maximum $-10 \mathrm{~dB}$ bandwidth is obtained with overlap of $1 \mathrm{~mm}$. Though this overlap size is the most suitable for this design, it may be not applicable for any design. Therefore, if such fixed overlap sizes are used, it is necessary to do several trial simulations with different overlap sizes to find the best size. The question here is whether it is possible to add an extra degree of freedom by letting the GA design an MPA with nonuniform overlaps. The novel method is proposed next.

3.3. Nonuniform Overlapping. It is found out whether the bandwidth can be increased further by using nonuniform overlaps. GA is used to assign different overlap sizes to each overlapping position. In this regard, any overlap size out of $0.5 \mathrm{~mm}, 1 \mathrm{~mm}$, and $2 \mathrm{~mm}$ is used at each position. This allows having conducting or nonconducting cells with different sizes instead of fixed sizes as used earlier. The optimized design 


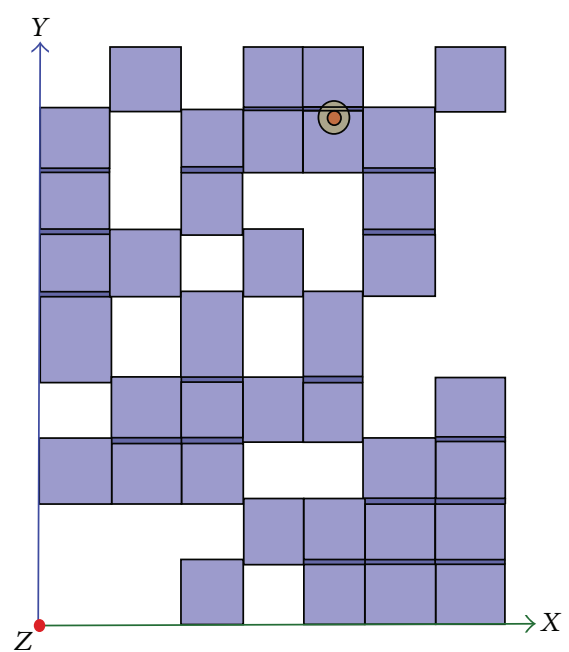

(a)

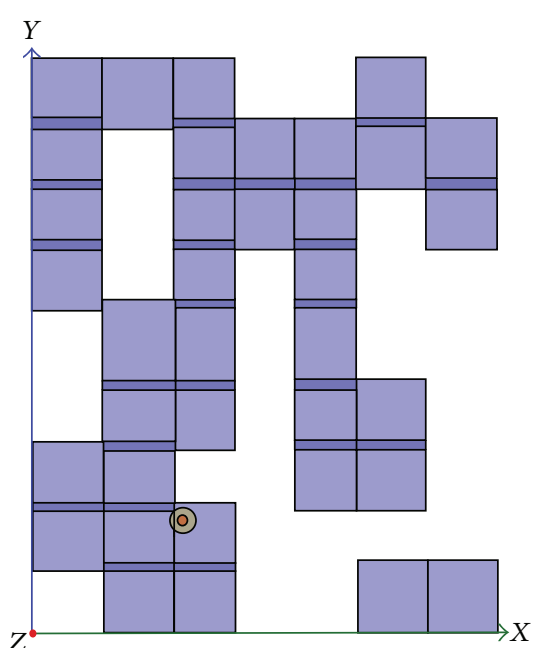

(b)

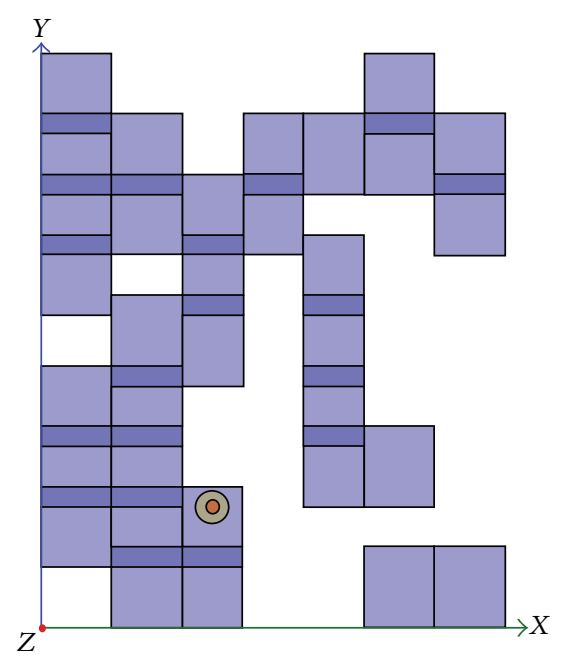

(c)

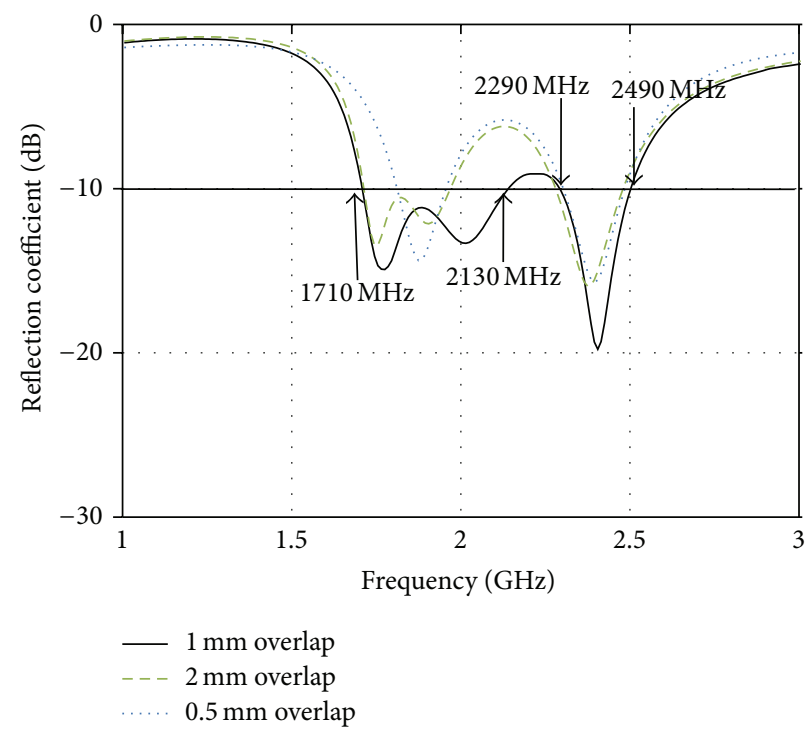

(d)

Figure 4: The optimized MPAs with uniform overlaps. (a) Optimized design for overlap of $0.5 \mathrm{~mm}$. (b) Optimized design for overlap of $1 \mathrm{~mm}$. (c) Optimized design for overlap of $2 \mathrm{~mm}$. (d) Reflection coefficient.

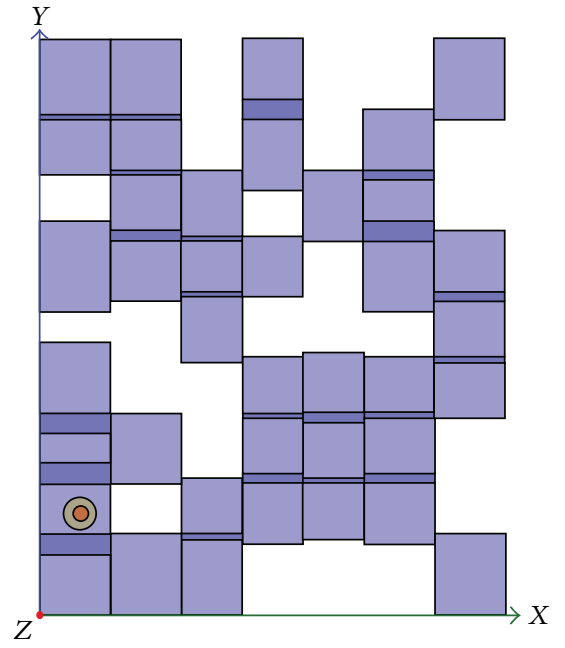

(a)

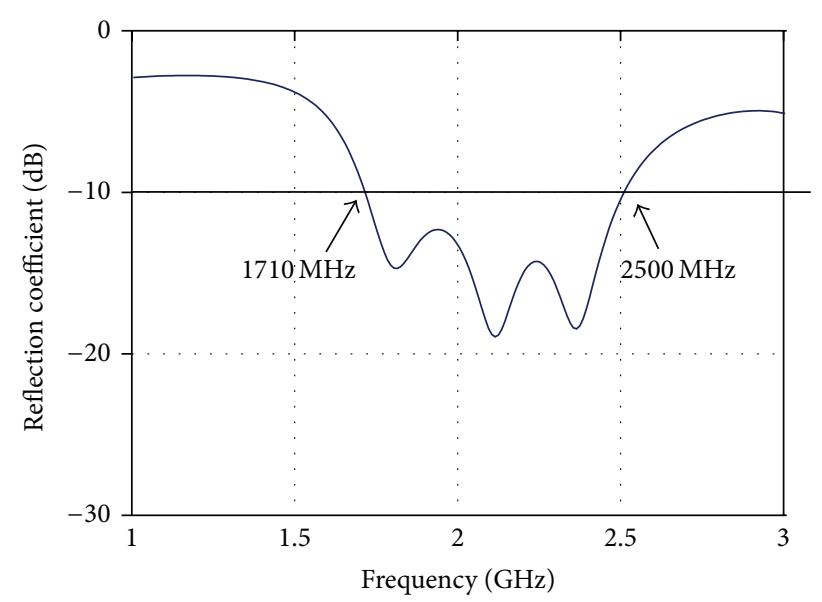

(b)

FIgURE 5: The optimized MPA with nonuniform overlaps. (a) Optimized design. (b) Reflection coefficient. 


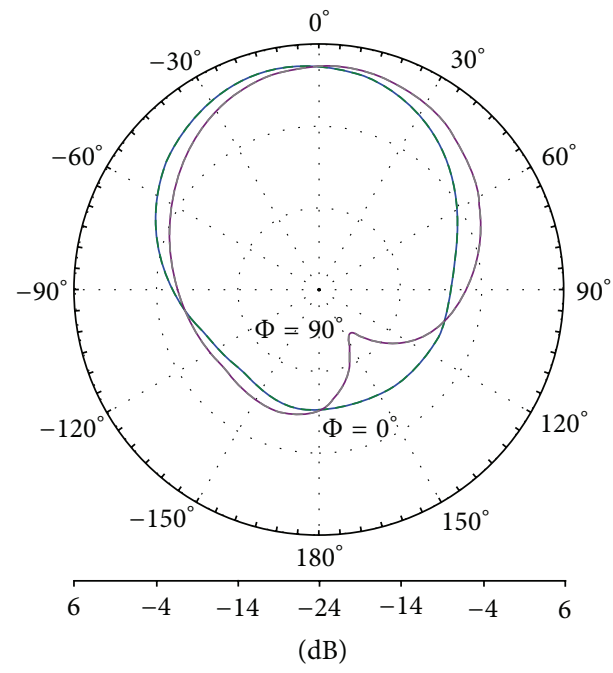

(a)

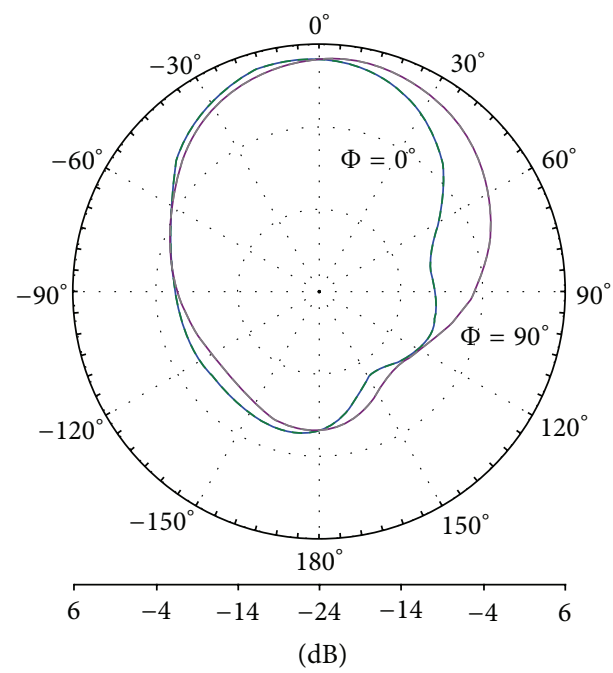

(c)

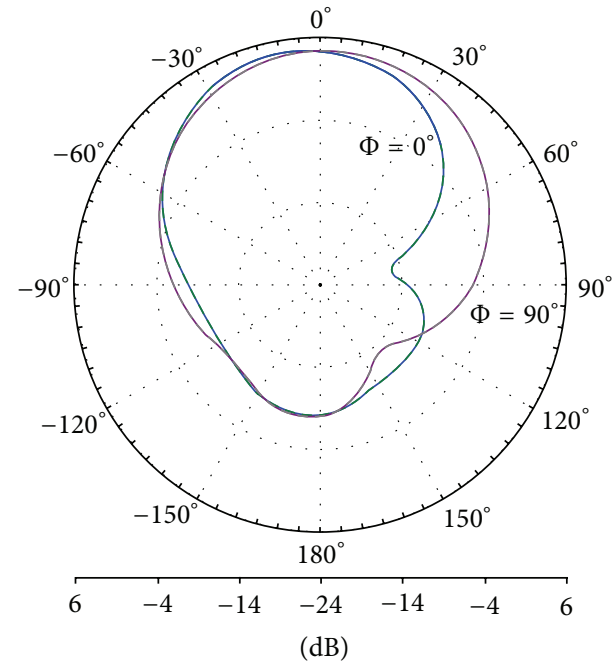

(b)

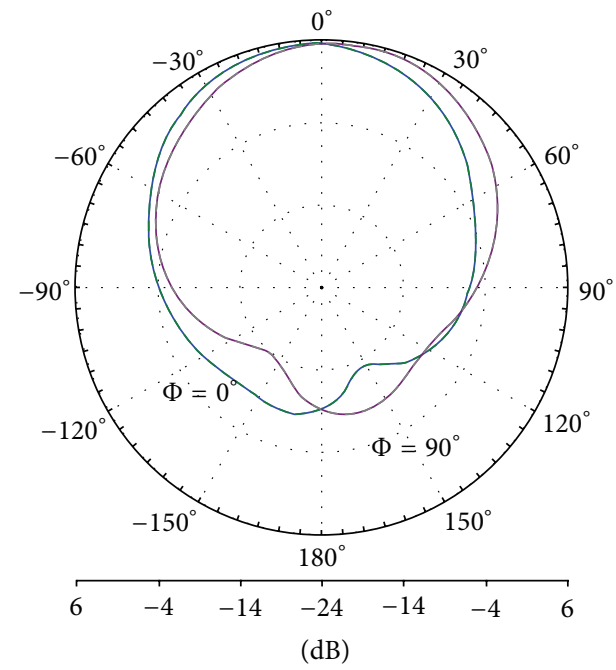

(d)

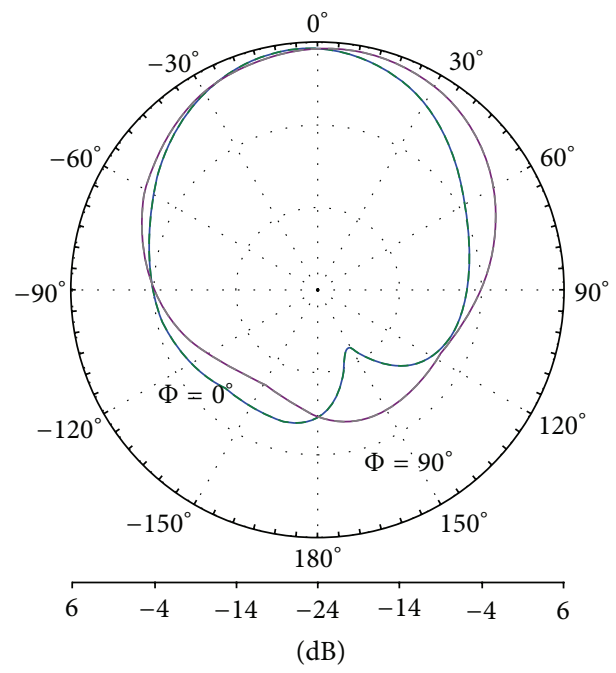

(e)

FIGURE 6: Radiation patterns at the center of each band. (a) GSM1800 ( $f=1800 \mathrm{MHz})$. (b) GSM1900 $(f=1920 \mathrm{MHz})$. (c) UMTS $(f=$ $2040 \mathrm{MHz}$ ). (d) LTE2300 ( $f=2350 \mathrm{MHz})$. (e) Bluetooth $(f=2440 \mathrm{MHz})$. 


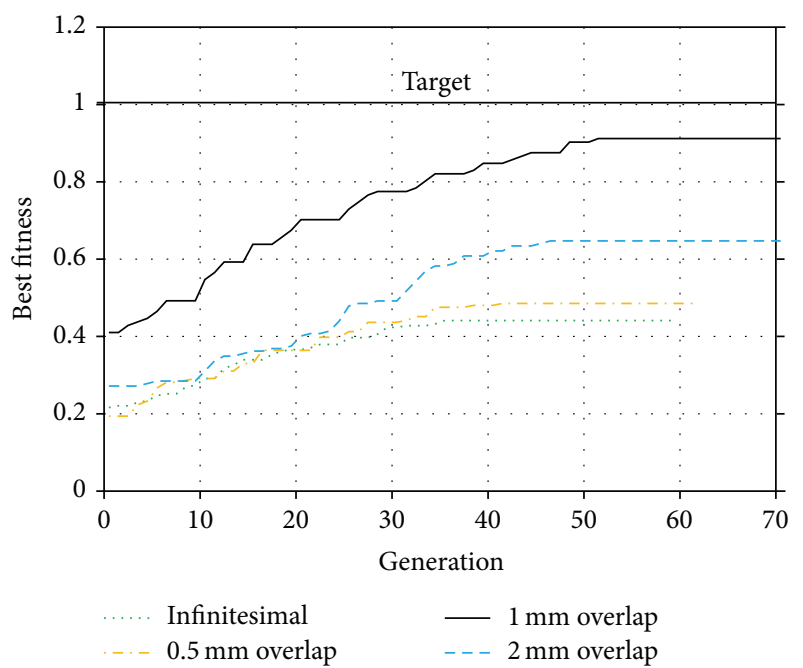

(a)

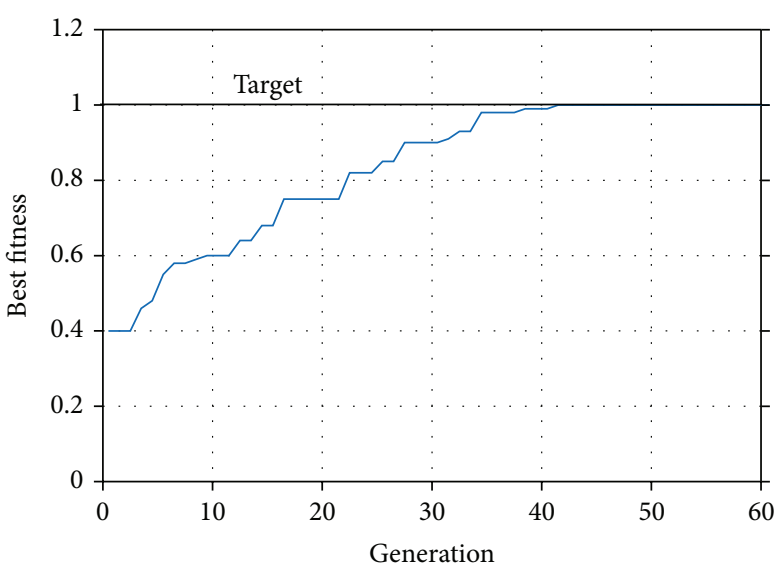

(b)

FIGURE 7: Best fitness over generations. (a) For infinitesimal connections and uniform overlaps. (b) For nonuniform overlaps.

consists of combination of different overlap sizes, resulting in a larger bandwidth from $1710 \mathrm{MHz}$ to $2500 \mathrm{MHz}$ (Figure 5). This pentaband design covers GSM1800, GSM1900, UMTS, LTE2300, and Bluetooth bands. Therefore, it seems that adding an extra flexibility in the overlapping between cells offers a better solution. The proposed antenna radiates along the broadside direction having gained values of $3.5 \mathrm{~dB}$, $4.6 \mathrm{~dB}, 4.3 \mathrm{~dB}, 5.6 \mathrm{~dB}$, and $5.2 \mathrm{~dB}$ at the center of GSM1800, GSM1900, UMTS, LTE2300, and Bluetooth bands, respectively (Figure 6). Compared to the radiation pattern of a conventional MPA, the optimized design has a lower gain at the lower frequency bands. However, if a higher gain is required, it can be achieved by modifying the fitness function accordingly. The radiation efficiency of this MPA is $96 \%$.

\section{Analysis of the Results}

As per the results, use of traditional on/off building blocks method has a low probability of creating a high performance MPA, in addition to fabrication problems. Use of overlaps between adjacent cells improves the performance, but the performance depends on the overlap size. However, the most appropriate overlap size changes from design to design and consumes much time for several trial simulations. As a solution, use of nonuniform overlapping is proposed in this paper and GA finds the best patch geometry with improved performance more effectively.

Variations of the best fitness over generations in the optimized designs are shown in Figure 7(a). The fitness values converge without reaching the target when the cells have infinitesimal connections or fixed overlap sizes. When nonuniform overlaps are used, the patch consists of conducting or nonconducting regions with different sizes. It adds more flexibility to the design and its fitness reaches the target after about 40 generations (Figure 7(b)). Therefore, this method helps to find the optimized design consuming less time.

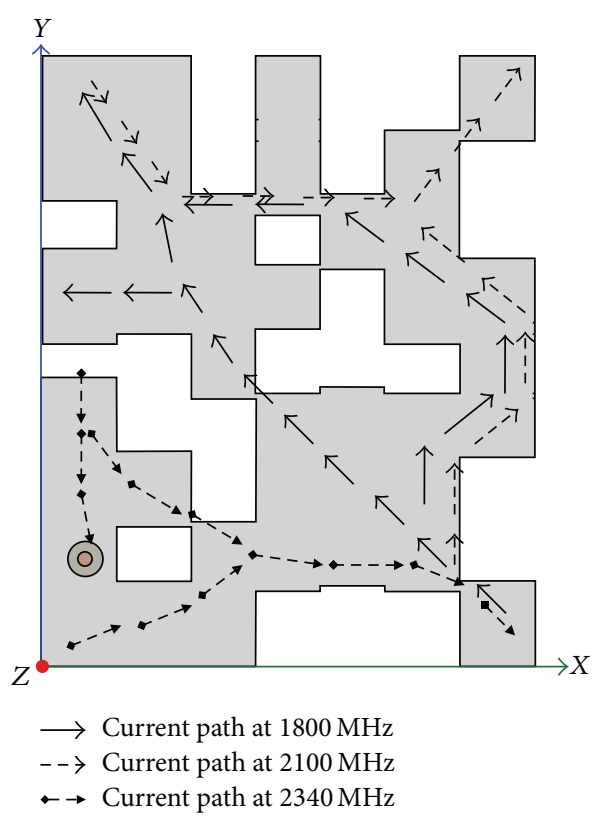

Figure 8: Current paths at resonant frequencies.

The optimized design with nonuniform overlapping resonates at three frequencies $(1800 \mathrm{MHz}, 2100 \mathrm{MHz}$, and $2340 \mathrm{MHz}$ ) within the required frequency band. As the resonant frequencies are close to each other, the resonant bands overlap giving the MPA multifrequency broadband performance. The current patterns at these three frequencies follow different paths resulting in a different resonant behavior compared to a classical rectangular patch (Figure 8). Current distribution patterns show that different areas of the patch are excited at three resonant frequencies (Figure 9).

This concept can be applied to the design of MPAs for different applications. For example, miniaturized MPAs can 


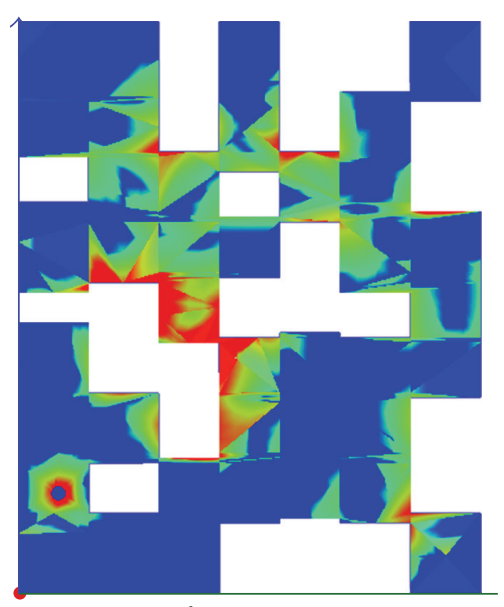

At $f=1800 \mathrm{MHz}$

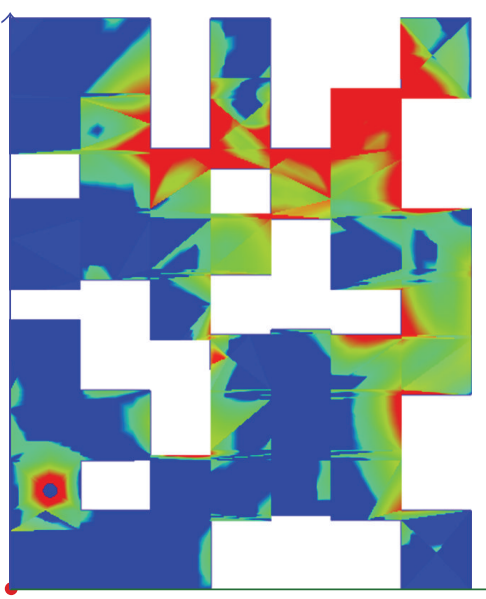

$f=2100 \mathrm{MHz}$

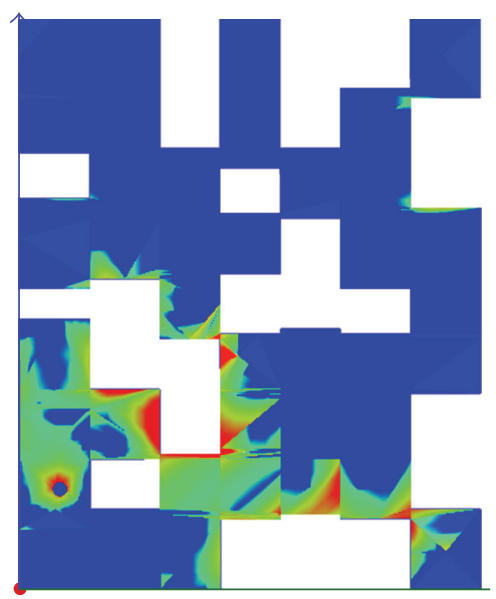

$f=2340 \mathrm{MHz}$

FIGURE 9: Current distribution at resonant frequencies.

be designed by employing techniques such as shorting pins and walls along with this concept. Such a shorted miniature MPA with same patch size and with uniform overlaps is presented in [11] by same authors. The reliability of simulation results has been confirmed by measurements.

\section{Conclusion}

This paper proposes a novel nonuniform overlapping method to improve the performance of MPAs by means of bandwidth, without increasing the antenna volume. It is based on nonuniformly shifting the cells parallel to the width of the MPA to avoid cells contacting each other by infinitesimal points. As this method gives an extra degree of freedom to the designer, the bandwidth of the MPA could be improved more easily compared to the fixed overlapping method. A pentaband design covering GSM1800, GSM1900, UMTS, LTE2300, and Bluetooth bands has been presented in this paper using this method. This nonuniform overlapping cell method can also be employed for other optimization problems such as the design of small and high-directivity MPAs.

\section{Conflict of Interests}

The authors declare that there is no conflict of interests regarding the publication of this paper.

\section{References}

[1] J. M. Johnson and Y. Rahmat-Samii, "Genetic algorithms in engineering electromagnetics," IEEE Antennas and Propagation Magazine, vol. 39, no. 4, pp. 7-21, 1997.

[2] R. L. Haupt, "Introduction to genetic algorithms for electromagnetics," IEEE Antennas and Propagation Magazine, vol. 37, no. 2, pp. 7-15, 1995.

[3] H. Choo, A. Hutani, L. C. Trintinalia, and H. Ling, "Shape optimization of broadband microstrip antennas using genetic algorithm," Electronics Letters, vol. 36, no. 25, pp. 2057-2058, 2000 .

[4] M. John and M. J. Ammann, "Design of a wide-band printed antenna using a genetic algorithm on an array of overlapping sub-patches," in Proceedings of the IEEE International Workshop on Antenna Technology Small Antennas and Novel Metamaterials, pp. 92-95, IEEE, March 2006.

[5] J. M. J. W. Jayasinghe and D. N. Uduwawala, "Optimization of the performance of patch antennas using genetic algorithms," Journal of the National Science Foundation of Sri Lanka, vol. 41, no. 2, pp. 113-120, 2013.

[6] L. A. Griffiths, C. Furse, and Y. C. Chung, "Broadband and multiband antenna design using the genetic algorithm to create amorphous shapes using ellipses," IEEE Transactions on Antennas and Propagation, vol. 54, no. 10, pp. 2776-2782, 2006.

[7] N. Herscovici, M. F. Osorio, and C. Peixeiro, "Miniaturization of rectangular microstrip patches using genetic algorithms," IEEE Antennas and Wireless Propagation Letters, vol. 1, pp. 94-97, 2002.

[8] M. Polivka, M. Drahovzal, J. Rohan, and P. Hazdra, "Multiband patch antenna with perturbation elements generated by genetic algorithm," in Proceedings of the European Conference on Antennas and Propagation (EuCAP '06), 4, p. 1, Nice, France, November 2006.

[9] O. Ozgun, S. Mutlu, M. I. Aksun, and L. Alatan, "Design of dual-frequency probe-fed microstrip antennas with genetic optimization algorithm," IEEE Transactions on Antennas and Propagation, vol. 51, no. 8, pp. 1947-1954, 2003.

[10] M. Ohira, H. Deguchi, M. Tsuji, and H. Shigesawa, "Multiband single-layer frequency selective surface designed by combination of genetic algorithm and geometry-refinement technique," IEEE Transactions on Antennas and Propagation, vol. 52, no. 11, pp. 2925-2931, 2004.

[11] J. W. Jayasinghe, J. Anguera, and D. N. Uduwawala, "A simple design of multi band microstrip patch antennas robust to fabrication tolerances for GSM, UMTS, LTE, and Bluetooth applications by using genetic algorithm optimization," Progress In Electromagnetics Research M, vol. 27, pp. 255-269, 2012. 
[12] P. Soontornpipit, C. M. Furse, and Y. C. Chung, "Miniaturized biocompatible microstrip antenna using genetic algorithm," IEEE Transactions on Antennas and Propagation, vol. 53, no. 6, pp. 1939-1945, 2005.

[13] J. Jayasinghe, J. Anguera, and D. Uduwawala, "Genetic algorithm optimization of a high-directivity microstrip patch antenna having a rectangular profile," Radioengineering, vol. 22, no. 3, pp. 700-707, 2013. 

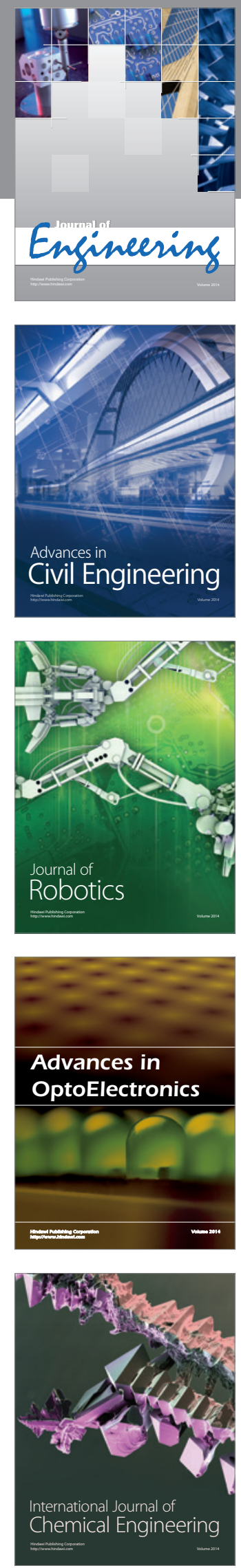

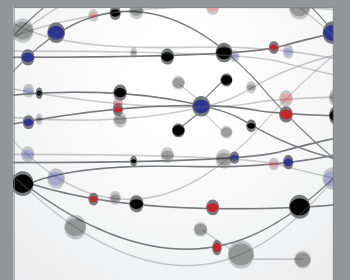

The Scientific World Journal
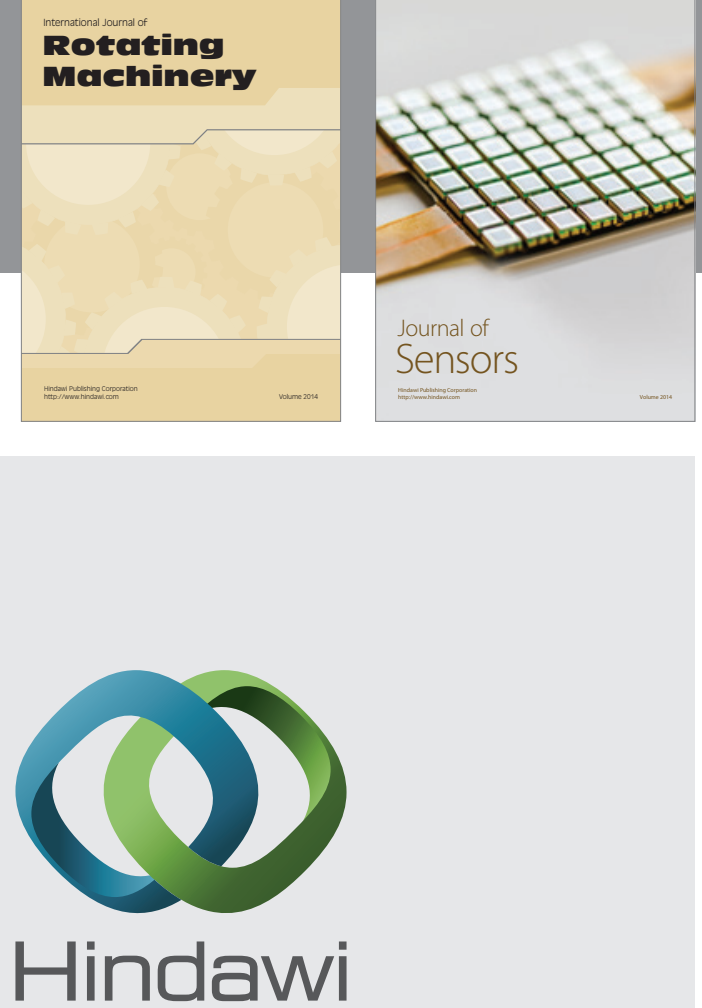

Submit your manuscripts at http://www.hindawi.com
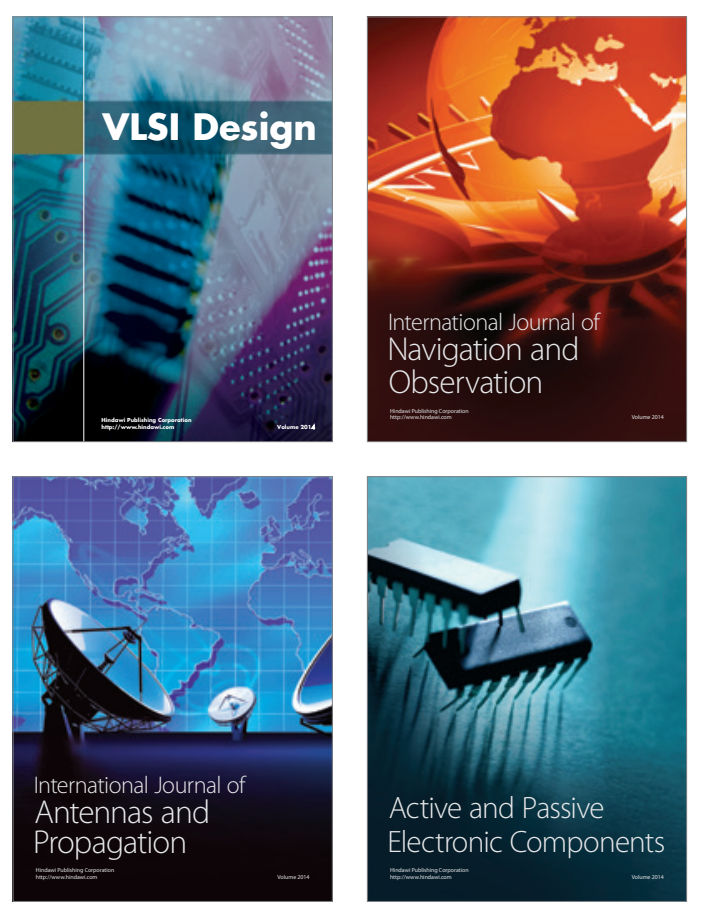
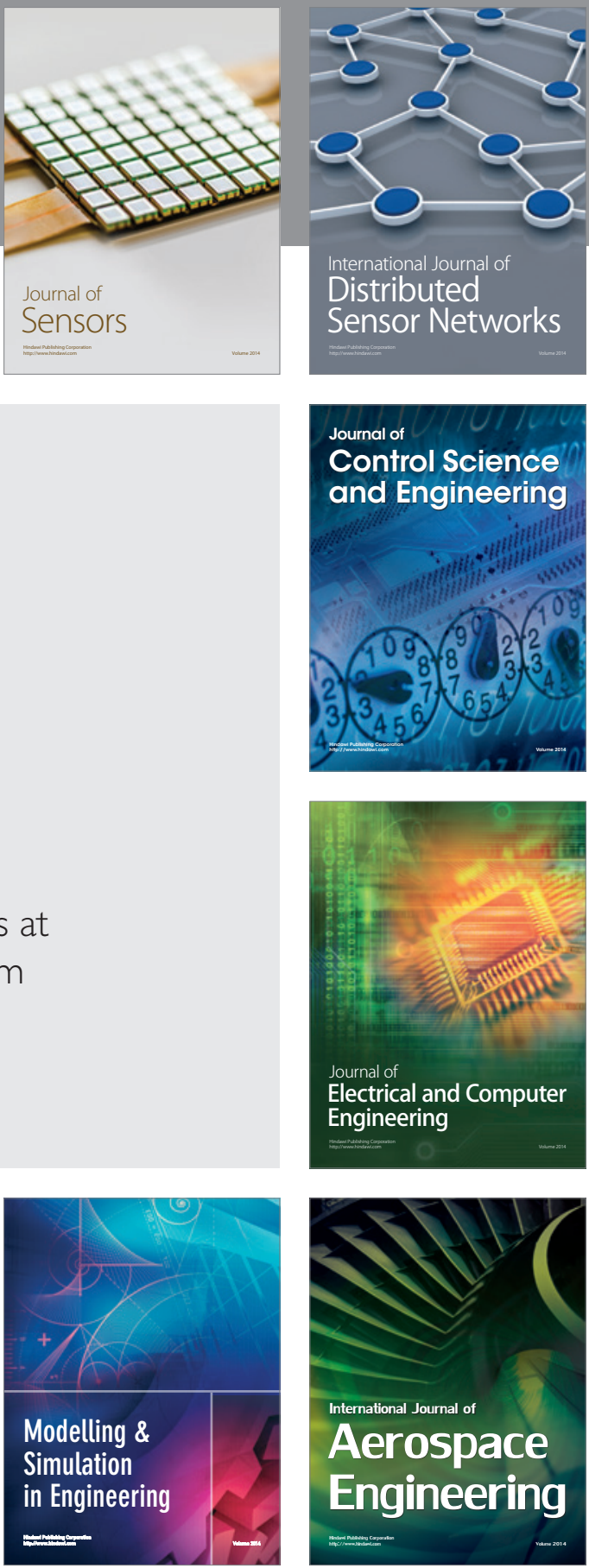

Journal of

Control Science

and Engineering
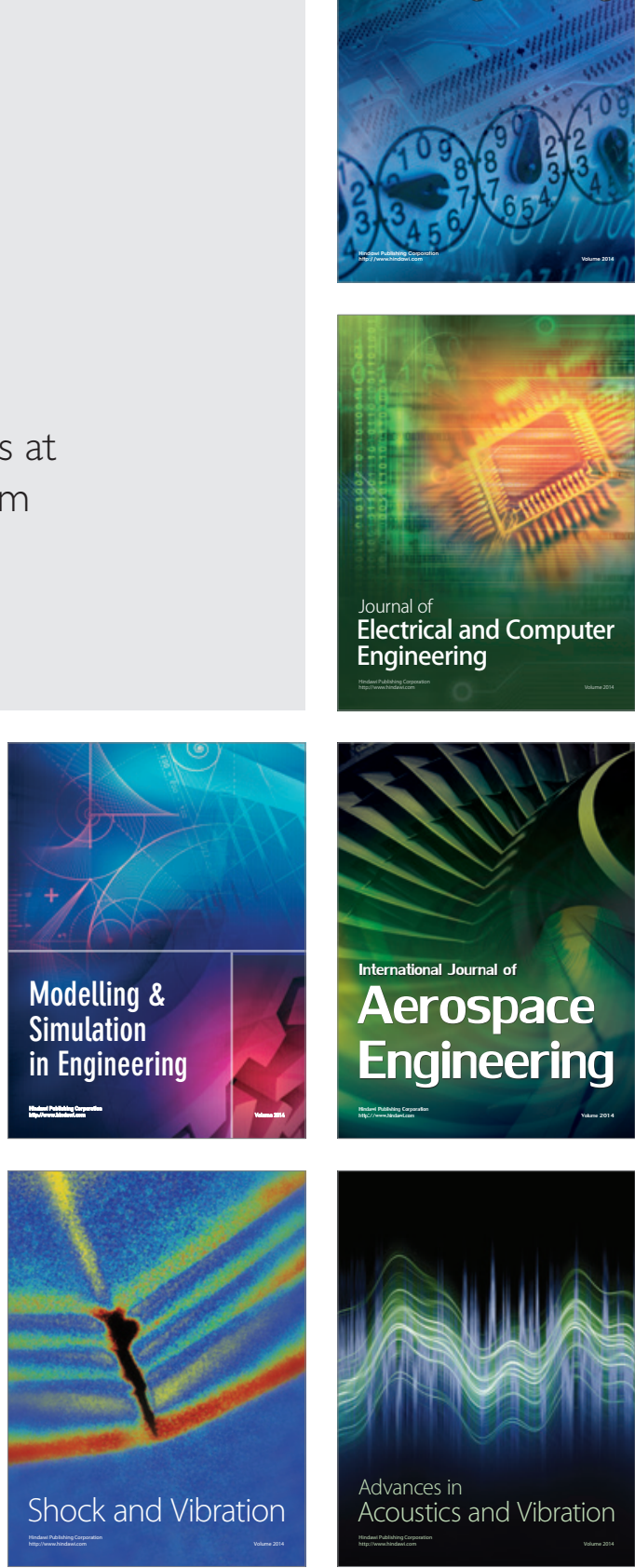